\title{
Euler graphs, triangle-free graphs and bipartite graphs in switching classes
}

\author{
Jurriaan Hage ${ }^{1}$, Tero Harju ${ }^{2}$, and Emo Welzl ${ }^{3}$ \\ 1 Inst. of Information and Computing Sci., Univ. Utrecht, P.O.Box 80.089, 3508 TB \\ Utrecht, Netherlands, jur@cs.uu.nl \\ 2 Dept. of Mathematics, University of Turku, FIN-20014 Turku, Finland \\ ${ }^{3}$ Institut für Theoretische Informatik, ETH Zentrum, IFW B49.2, Haldeneggsteig 4, \\ CH - 8092 Zürich, Switzerland
}

\begin{abstract}
In the context of graph transformations we look at the operation of switching, which can be viewed as an elegant method for realizing global transformations of graphs through local transformations of the vertices. A switching class is then a set of graphs obtainable from a given start graph by applying the switching operation.

Continuing the line of research in Ehrenfeucht et al. we consider the problem of detecting three kinds of graphs in switching classes. For all three we find algorithms running in time polynomial in the number of vertices in the graphs, although switching classes contain exponentially many graphs.
\end{abstract}

\section{Introduction}

The operation of switching is an elegant example of a graph transformation, where the global transformation of a graph is achieved by applying local transformations to the vertices. The elegance stems from the fact that the local transformations are group actions, and hence basic techniques of group theory can be applied in developing the theory of switching.

For a finite undirected graph $G=(V, E)$ and a subset $\sigma \subseteq V$, the switch of $G$ by $\sigma$ is defined as the graph $G^{\sigma}=\left(V, E^{\prime}\right)$, which is obtained from $G$ by removing all edges between $\sigma$ and its complement $V-\sigma$ and adding as edges all nonedges between $\sigma$ and $V-\sigma$. The switching class $[G]$ determined (generated) by $G$ consists of all switches $G^{\sigma}$ for subsets $\sigma \subseteq V$.

A switching class is an equivalence class of graphs under switching, see the survey papers by Seidel [11] and Seidel and Taylor [12]. Generalizations of this approach - where the graphs are labelled with elements of a group other than $\mathbf{Z}_{2}$ - can be found in Gross and Tucker [6], Zaslavsky [13], the book of Ehrenfeucht, Harju and Rozenberg [5] and the thesis of Hage [7]. 
A property $\mathcal{P}$ of graphs can be transformed into an existential property of switching classes as follows:

$\mathcal{P}_{\exists}(G)$ if and only if there is a graph $H \in[G]$ such that $\mathcal{P}(H)$.

We will also refer to $\mathcal{P}_{\exists}$ as "the problem $\mathcal{P}$ for switching classes".

Let $G$ be a graph on $n$ vertices. It is easy to show that there are $2^{n-1}$ graphs in $[G]$, and so checking whether there exists a graph $H \in[G]$ satisfying a given property $\mathcal{P}$ requires exponential time, if each graph is to be checked separately.

However, we prove that deciding that a switching class is bipartite (i.e., it contains at least one bipartite graph) can be done in polynomial time. The same result holds for the predicates "triangle-free" and "eulerian". The solution to the latter problem can also be found in the thesis of Hage [7], but has never been published in a paper.

A little now about the motivation for our research. In [8], we considered the problem of characterizing the switching classes which contain only cyclic graphs. We did this by means of a list of forbidden subgraphs. In the process of this research we made extensive use of computer programs. However, as stated earlier, switching classes grow unwieldy quite soon and having an efficient algorithm to check whether a switching class contains any acyclic graphs at all, would be of great help. For the case of acyclic graphs such an algorithm exists (Theorem 1 of Ehrenfeucht, Hage, Harju and Rozenberg [4] or Theorem 3.21 of Hage [7]).

After considering this problem for acyclic graphs we turned to generalizations of the acyclic graphs: the bipartite and the triangle-free graphs. The bipartite graphs and the triangle-free graphs are natural classes of graphs in the context of switching, see Seidel [11] and Seidel and Taylor [12]. In the latter case, it seems that there is not a reasonably elegant result using a list of forbidden subgraphs: this list soon grows to enormous size. For the bipartite case however the computational results so far are promising.

The computational complexity of various problems for switching classes were considered by Ehrenfeucht, Hage, Harju and Rozenberg [4]. In the present paper we continue this approach by showing that the three problems to decide whether a switching class contains a bipartite respectively eulerian respectively triangle-free graph are all polynomial time (in the number of vertices of the graphs). Although we consider graphs up to equality (i.e. the names of the vertices do matter), most results carry over to the situation where the graphs are considered up to isomorphism. 


\section{Preliminaries}

For a (finite) set $V$, let $|V|$ be the cardinality of $V$. We shall often identify a subset $A \subseteq V$ with its characteristic function $A: V \rightarrow \mathbf{Z}_{2}$, where $\mathbf{Z}_{2}=$ $\{0,1\}$ is the cyclic group of order two. We use the convention that for $x \in V, A(x)=1$ if and only if $x \in A$. The restriction of a function $f: V \rightarrow W$ to a subset $A \subseteq V$ is denoted by $\left.f\right|_{A}$. We denote set difference by $A-B$. It contains the elements in $A$ which are not in $B$. If $B$ is a singleton $\{b\}$, then we may write $A-b$ for brevity.

The set $E(V)=\{\{x, y\} \mid x, y \in V, x \neq y\}$ denotes the set of all unordered pairs of distinct elements of $V$. We write $x y$ or $y x$ for the undirected pair $\{x, y\}$. The graphs of this paper will be finite, undirected and simple, i.e., they contain no loops or multiple edges. We use $E(G)$ and $V(G)$ to denote the set of edges $E$ and the set of vertices $V$, respectively, and $|V|$ and $|E|$ are called the order, respectively, size of $G$. Analogously to sets, a graph $G=(V, E)$ will be identified with the characteristic function $G: E(V) \rightarrow \mathbf{Z}_{2}$ of its set of edges so that $G(x y)=1$ for $x y \in E$, and $G(x y)=0$ for $x y \notin E$. Later we shall use both notations, $G=(V, E)$ and $G: E(V) \rightarrow \mathbf{Z}_{2}$, for graphs.

To establish notation and terminology we repeat some elements of graph theory.

Let $G=(V, E)$ be a graph. A vertex $x \in V$ is adjacent to $y \in V$ if $x y \in E$. The degree of $x$ in $G$ is the number of vertices it is adjacent to. The neighbours of $u$ in $G$, denoted $N_{G}(u)$, or $N(u)$ if $G$ is clear from the context, is the set of vertices adjacent to $u$ in $G$. A vertex which is not adjacent to any other vertex in a graph is called isolated.

For a graph $G=(V, E)$ and $X \subseteq V$, let $\left.G\right|_{X}$ denote the subgraph of $G$ induced by $X$. Hence, $\left.G\right|_{X}: E(X) \rightarrow \mathbf{Z}_{2}$. For two graphs $G$ and $H$ on $V$ we define $G+H$ to be the graph such that $(G+H)(x y)=G(x y)+H(x y)$ for all $x y \in E(V)$.

Some graphs we will encounter in the sequel are $K_{V}$, the clique on the set of vertices $V$, and $\bar{K}_{V}$, the complement of $K_{V}$ which is the discrete graph on $V$; the complete bipartite graph on $A$ and $V-A$ is denoted by $K_{A, V-A}$. If the choice of vertices is unimportant we can write $K_{n}, \bar{K}_{n}$ and $K_{m, n-m}$ for $n=|V|$ and $m=|A|$.

An euler graph is a connected graph consisting of a single closed walk such that each edge is traversed once (but a vertex can be visited several times). It is well-known that a graph is eulerian if and only if all the degrees of its vertices are even. We can extend the definition of euler graphs to disconnected graphs by demanding that all components are 
eulerian. These graphs are here called even graphs. In a similar fashion we call graphs in which every vertex has odd degree an odd graph.

A graph $G=(V, E)$ is bipartite if $V$ can be partitioned into two sets $A$ and $V-A$, such that all edges in $E$ are between $A$ and $V-A$. An equivalent characterization is to say that $G$ is bipartite if and only if it contains no cycles of odd length. Deciding whether a graph is bipartite is easily done by visiting each vertex at most once. So it can be checked for graphs very efficiently. The bipartite graphs include among them all acyclic graphs, because these do not contain cycles of any kind.

The triangle-free graphs generalize bipartite graphs by only disallowing triangles: a graph $G=(V, E)$ is triangle-free if $G$ does not contain an induced $K_{3}$. Note that if $G$ is triangle-free, then its largest clique (i.e., complete subgraph) has at most two vertices. Deciding whether a graph is triangle-free is easily done by examing every subset of three vertices to see if all three edges in the triangle are in the graph.

Triangle-freeness can be decided in time cubic in the number of vertices. Whether a graph is eulerian and bipartite can both be decided in time quadratic in the number of vertices of the graph. In the following we shall consider all three problems for switching classes and show that the problems remain polynomial.

We continue now with definitions for the switching of graphs.

A selector for $G$ is a subset $\sigma \subseteq V(G)$, or alternatively a function $\sigma: V(G) \rightarrow \mathbf{Z}_{2}$. A switch of a graph $G$ by $\sigma$ is the graph $G^{\sigma}$ such that for all $x y \in E(V)$,

$$
G^{\sigma}(x y)=\sigma(x)+G(x y)+\sigma(y) .
$$

Clearly, this definition of switching is equivalent to the one given at the beginning of the introduction. The set $[G]=\left\{G^{\sigma} \mid \sigma \subseteq V\right\}$ is called the switching class of $G$. We reserve lower case $\sigma$ for selectors (subsets) used in switching.

A selector $\sigma$ is constant on $X \subseteq V$ if $X \subseteq \sigma$, or $X \cap \sigma=\emptyset$. The name arises from the fact that, in these cases, $\left.G\right|_{X}=\left.G^{\sigma}\right|_{X}$.

We now give a few results from the literature that will be used throughout this paper, see Seidel [11], Ehrenfeucht, Harju and Rozenberg [5] and Hage [7].

Lemma 1. The switching class $\left[\bar{K}_{V}\right]$ equals the set of all complete bipartite graphs on $V$.

Proof. Given any complete bipartite graph $K_{\{\sigma, V-\sigma\}}$, we can obtain it from $\bar{K}_{V}$ by switching with respect to $\sigma$. Also, every switch of $\bar{K}_{V}$ can 
divide its partition at most in two, so it must be a bipartite graph. It is obviously complete bipartite, because $\bar{K}_{V}$ has no edges.

From the observation that applying a selector $\sigma$ to $G$ amounts to computing $G+K_{\sigma, V(G)-\sigma}$ we obtain the following result.

Lemma 2. It holds that $G \in[H]$ if and only if $G+H \in\left[\bar{K}_{V}\right]$.

Lemma 3. Let $G=(V, E)$ be a graph, $u \in V$ and $A \subseteq V-\{u\}$. There exists a unique graph $H \in[G]$ such that the neighbours of $u$ in $H$ are the vertices in $A$.

Proof. The vertex $u$ has no adjacent vertices in $G_{u}=G^{N(u)}$, where $N(u)$ is the set of neighbours of $u$ in $G$. Switching $G_{u}$ with respect to $A$ connects $u$ to every vertex in $A$ (and no others) yielding $H$.

To show that $H$ is unique: let $H^{\prime}$ be such that $N(u)=A$ in $H^{\prime}$. Since $H$ and $H^{\prime}$ are in the same switching class $H+H^{\prime}$ is a complete bipartite graph (Lemma 2), say $G_{B, V-B}$. Because $u$ has the same neighbours in both, $u$ is isolated in $G_{B, V-B}$. Hence, $G_{B, V-B}$ is a discrete graph and, consequently, $H=H^{\prime}$.

As a corollary we easily find that for every vertex $x \in V(G)$, there is a unique graph in $[G]$ where $x$ is isolated.

\section{$3 \quad$ Euler switching classes}

Let $G$ be a graph. Recall that $G$ is called an even (odd) graph if all vertices are of even (odd) degree.

Seidel [10] proved that if $G$ is of odd order, then the switching class $[G]$ contains a unique even graph. Such a result is interesting because it tells us that every graph having an odd number of vertices can be constructed from an even graph using the rather simple transformation of switching. (See also Mallows and Sloane [9] and Cameron [2] for the connection of eulerian graphs to switching.)

Theorem 1 (Seidel [10]). Let $G$ be a graph of odd order. Then $[G]$ contains a unique even graph.

Proof. Let $G$ be a graph of odd order. The even graph can be obtained by switching with respect to the set $\sigma$ of vertices in $G$ that have odd degree, because there are an odd number of vertices of even degree and an even number of vertices of odd degree. For instance, if a vertex $v$ 
had odd degree and an even number of its neighbours had even degree, then $v$ loses an even number of neighbours, but gains an odd number of neighbours, since the number of vertices of even degree is odd. In sum, $v$ will have an even number of neighbours in $G^{\sigma}$. The other three cases can be treated in a similar way. Hence $G^{\sigma}$ has only vertices of even degree.

Let $H \in[G]$ be an even graph on vertices $V$ and $\tau$ a nontrivial selector so that $H^{\tau}$ is also even. We can assume without loss of generality that $\tau$ has even cardinality. Let $v \in \tau$. We can split $V-\{v\}$ into four sets of vertices based on the fact whether a vertex is connected to $v$ or not and the fact whether they are or are not in $\tau$. The vertex $v$ will be connected to all vertices in $A=(V-\tau) \cap\left(V-N_{H}(v)\right)$, i.e., the vertices outside $\tau$ that it was not connected to, and $B=\tau \cap N_{H}(v)$, i.e., the neighbours it was connected to. We now show $|A \cup B|$ is odd, which implies that $H^{\tau}$ is not even. Assume $|B|$ is odd. Then $\left|(V-\tau) \cap N_{H}(v)\right|$ is odd, because $v$ was even. Because $|B \cup\{v\}|$ is even and $|\tau|$ is even, we find that $|\tau-(B \cup\{v\})|$ is even. It follows that $|A|$ is even, because the order of $H$ is odd. Similar reasoning for the case that $|B|$ is even proves that $H$ can not be switched into an even graph, unless the switch is trivial, which proves that the even graph $H$ is unique.

A general uniqueness result such as Theorem 1 is not possible without restrictions on the vertex set. Indeed, if $\mathcal{P}$ is any graph property, which is preserved under isomorphisms, then there exists a switching class that has no graphs with $\mathcal{P}$ or that has at least two graphs with $\mathcal{P}$. This statement follows from a result on automorphisms of switching classes, see Cameron [2]: there exist switching classes where the first cohomological invariant is different from zero, and therefore there exist switching classes for which the group of automorphisms is strictly larger than the group of automorphisms of its graphs.

For graphs of even order, a switching class $[G]$ can contain only noneulerian graphs, e.g. take the switching class $\left[P_{4}\right]$. However, it holds that either $[G]$ has no even and no odd graphs, or exactly half of its graphs are even while the other half are odd, as first proved in Ehrenfeucht, Hage, Harju and Rozenberg [4]. (This is a result that is not likely to hold when graphs are considered up to isomorphism.)

To see this, define $u \sim_{G} v$, if $d_{G}(u) \equiv d_{G}(v)(\bmod 2)$, that is, if the degrees of $u$ and $v$ have the same parity. This relation is an equivalence relation on $V(G)$.

Assume then that the order $n$ of $G$ is even. If we consider singleton selectors $\sigma$ only (hence switching with respect to one vertex), then it is easy to see that $\sim_{G}$ and $\sim_{G^{\sigma}}$ coincide for all selectors $\sigma$. In other words, 
if $G$ has even order, then the relation $\sim_{G}$ is an invariant of the switching class $[G]$. This means that if $[G]$ contains an even graph, then all graphs in $[G]$ are either even or odd. Moreover, if $G$ is even, and $\sigma: V(G) \rightarrow \mathbf{Z}_{2}$ is a singleton selector, then for each $v \in V(G), d_{G}(v)$ and $d_{G^{\sigma}}(v)$ have different parity.

Theorem 2. Let $G$ be a graph of even order and such that $[G]$ contains an even graph. Then $[G]$ contains an eulerian graph unless $[G]$ contains a complete graph.

Proof. We may assume that $G$ itself is even, otherwise switch at an arbitrary vertex. However, this graph may not be connected and we need to find a connected even switch of $G$. This can be done as follows: let $u$ be any vertex of $G$. Let $V=V(G)$ and let $O=V-\left(\{u\} \cup N_{G}(u)\right)$, the vertices that are not neighbours of $u$ in $G$. Note that the set $O$ has odd cardinality. Switch $G$ with respect to $\sigma=O-\{v\}$ for an arbitrary $v \in O$. The resulting graph $G^{\sigma}$ is again even, but it may still not be connected, but at least $\left.G^{\sigma}\right|_{V-v}$ is. However, if $\left.G^{\sigma}\right|_{V-v}$ is not the complete graph, we can choose a vertex $w \in V-\{u, v\}$ that is not connected to all vertices in $V-v$. Switching with respect to $\{v, w\}$ now gives us a connected even graph. Note that in fact $\left[K_{n}\right]$ for even $n$ does have even graphs, but no eulerian graphs, because $K_{n}$ is the only connected graph and it is not even, but odd.

From the above it follows that the existence of an even graph in a switching class can be determined in time linear in the size of the graph: in the odd order case, the answer is always yes, while in the even order case, we need only verify whether $G$ is an even or odd graph. If either of these is the case, then the answer is yes, otherwise it is no.

We can extend this algorithm to check for eulerian graphs: for graphs $G$ of odd order it is simply a question of finding the unique even graph as in the proof Theorem 1 and verifying whether or not it is connected. For the even case we can use the algorithm implied in the proof of Theorem 2.

\section{Triangle-free switching classes}

The smallest graph that generates a switching class without bipartite graphs, also has no triangle-free graphs. It is the switching class $\left[K_{5}\right]$, which contains in addition to $K_{5}$ only $K_{4} \oplus K_{1}$ and $K_{3} \oplus K_{2}$, where $\oplus$ denotes the operation of disjoint union of two graphs. In this section we investigate the problem of deciding whether a switching class contains any triangle-free graphs. 
The algorithm is specified by means of a reduction to the 2SAT problem, a conjunction of disjunctions such that each disjunction contains at most two literals, where in our case each literal stands for a vertex in the graph. If the literal occurs positively this will mean that the vertex is in the selector corresponding to the instance of 2SAT. If it occurs negatively, it will not occur in this selector.

An example instance of this problem is $\bar{u} \wedge(u \vee v)$. A selector that satisfies this proposition does not select $u$, because of the first clause and hence must select $v$ to make the second clause true.

In what follows we shall omit $\wedge$ from the propositions by writing, e.g., $\bar{u}(u \vee v)$. We often use $\otimes$ for the xor connective. Recall that $x \otimes y$ is equivalent to $(x \vee y)(\bar{x} \vee \bar{y})$.

Theorem 3. Deciding whether or not a switching class contains a trianglefree graph can be decided time polynomial in the order of the graph.

Proof. We may assume, by Lemma 3, that $G$ contains an isolated vertex $v$ and we set $\sigma(v)=1$. Hence, our 2SAT formula contains the clause $v$. Note that our choice to select $v$ is no restriction, because a selector is equivalent to its complement.

If $G$ contains $K_{4}$, then we are done, because then we can conclude that $G$ does not have a triangle-free switch (take the $K_{4}$ with isolated vertex $v)$. Second, if the graph $G$ is triangle-free, then we can also stop. In the other case, $G$ contains a triangle, say $T$. Assume that $\sigma$ is the selector that maps $G$ into a triangle-free graph. Obviously, $\sigma$ is not constant on $T$. In this case only one degree of freedom remains: which single vertex $u \in T$ has $\sigma(u)=0$ ? If we had two vertices in $T$ with $\sigma\left(u_{1}\right)=\sigma\left(u_{2}\right)=0$, then $\left\{u_{1}, u_{2}, v\right\}$ would be a triangle in $G^{\sigma}$.

We now choose an arbitrary vertex $u \in T$ and set $\sigma(u)=0$ and $\sigma(x)=1$ for $x \in T-\{u\}$. All other neighbours of $u$ in $G$ must also be selected: if one of them, say $z$, was not, then $\{z, u, v\}$ would be a triangle in $G^{\sigma}$.

The foregoing can be summarized as follows: the selector $\sigma$ satisfies the formula

$$
\alpha_{G}(u)=v \bar{u} \bigwedge_{x \in N(u)} x
$$

Note that $\left.G\right|_{N(u)}$ can have no induced triangles, because then $G$ would contain an induced $K_{4}$, a case we have already taken care of.

The only remaining vertices are those in $M(u)=V(G)-(N(u) \cup$ $\{u, v\})$. Consider an edge $x y \in E\left(\left.G\right|_{M(u)}\right)$. If both of them or neither of 
them are selected by $\sigma$, then $G^{\sigma}$ contains either a triangle $\{x, y, u\}$ or $\{x, y, v\}$, depending on whether $\sigma(x)=1$ or $\sigma(x)=0$ respectively.

In other words we obtain

$$
\beta_{G}(u)=\alpha_{G}(u) \bigwedge_{x y \in E, x, y \in M(u)}(x \otimes y) .
$$

Note that this formula can only be satisfied if $\left.G\right|_{M(u)}$ is bipartite.

Let $\tau$ be any selector that satisfies $\beta_{G}(u)$. Then $G^{\tau}$ has the following properties:

i. $v$ is not in any triangle in $G^{\tau}$,

ii. $u$ is not in any triangle in $G^{\tau}$,

iii. $\left.G^{\tau}\right|_{N(u) \cup\{u, v\}}$ is triangle-free, and

iv. $\left.G^{\tau}\right|_{M(u)}$ is bipartite and hence triangle-free.

We have now come this far: if $\sigma$ satisfies $\beta_{G}(u)$, then the only triangles that can remain in $G^{\sigma}$ involve either

- two vertices from $N(u)$ and one from $M(u)$, or

- two vertices from $M(u)$ and one from $N(u)$.

We continue now with the prevention of triangles $\{x, y, z\}$ with $x \in$ $M(u)$ and $y, z \in N(u)$. We need to consider only triangles $\{x, y, z\}$ and the case that $G(y z)=1, G(x y)=0, G(x z)=0$. This follows from the fact that $\left.G\right|_{\{x, y, z\}}$ must be a switch of $K_{3}$, so it is either $K_{3}$ or an edge with an isolated vertex. In the latter case, there are three possibilities: the edge in question is $x y, x z$ or $y z$. In the former two cases, $y z$ will not be an edge in a switch $G^{\sigma}$ of $G$ that satisfies $\beta_{G}(u)$, because $\sigma$ is constant on $N(u)$. So in the end only the third case, $y z \in E(G)$ needs to be considered.

We define

$$
\begin{gathered}
X_{0}=\{x \in M(u) \mid \exists y, z \in N(u): G(y z)=G(x y)=G(x z)=1\}, \\
X_{1}=\{x \in M(u) \mid \exists y, z \in N(u): G(y z)=1, G(x y)=0, G(x z)=0\}
\end{gathered}
$$

and take as our improved formula

$$
\gamma_{G}(u)=\beta_{G}(u) \bigwedge_{x \in X_{1}} x \bigwedge_{x \in X_{0}} \bar{x} .
$$

The conjunction over $X_{0}$ forces all triangles involving one vertex from $M(u)$ and two from $N(u)$ to be removed from $G$. On the other hand, the conjunction over $X_{1}$ prevents the introduction of triangles of this kind 
after switching. It is possible for $X_{0} \cap X_{1}$ to be non-empty, but in that case the formula will not be satisfied by any selector.

For the final case, let $x, y \in M(u)$ and $z \in N(u)$. As we did earlier, we need only consider the case that the subgraph $\left.G\right|_{\{x, y, z\}}$ is a switch of $K_{3}$. Every edge in $\left.G\right|_{M(u)}$ will be destroyed by any selector that satisfies $\gamma_{G}(u)$. This immediately takes care of the case that $G(x y)=1$ (this includes the case that $\{x, y, z\}$ is a triangle in $G$ ).

The second case is when $G(x y)=0$. In that case either $G(x z)=0$ and $G(y z)=1$ or vice versa, $G(x z)=1$ and $G(y z)=0$. We assume the former. In that case, the only way in which we can possibly introduce a triangle is by $\sigma(x)=0$ and $\sigma(y)=1$. For this reason we add for all such pairs $x$ and $y$ the clause $x \vee \bar{y}$ to the formula $\gamma_{G}(u)$, obtaining in this way our final formula $\delta_{G}(u)$.

If there is no selector that satisfies $\delta_{G}(u)$, then we can construct the formula $\delta_{G}\left(u^{\prime}\right)$ for the other two vertices $u^{\prime} \in T-\{u\}$. If neither of these yield a formula that can be satisfied by a selector, then the switching class contains no triangle-free graphs: the choice of $T$ is an arbitrary one, because a selector must dissolve all existing triangles in $G$. The completeness of the algorithm then follows from the fact that the 2SAT formulas by construction only forbid selectors that are guaranteed to map into graphs with triangles.

Note that if $\delta_{G}(u)$ is satisfiable for some $u$, then we can easily construct the triangle-free graph by constructing the selector that satisfies the formula and applying it to $G$. We have shown that there are only three choices for $u$, and in all three cases, the formula has size at most quadratic in the number of vertices. 2SAT itself can be solved in time linear in the size of the formula [1] so we obtain a polynomial time complexity for our problem.

Example 1. We shall now give a small example, one which also indicates the need for trying all three vertices of a chosen triangle.

In Figure 1 we have depicted the same graph twice, with different choices for $u$. Choosing $u_{1}$ for $u$ does not give us a switch into a trianglefree graph (see Figure 1(a)). The reason is that the triangle $\left\{u_{2}, u_{3}, u_{6}\right\}$ must be removed (so $u_{6}$ must not be switched), but this induces a triangle $\left\{u_{4}, u_{5}, u_{6}\right\}$. We get a similar situation for $u=u_{3}$. However, if we choose $u=u_{2}$, then the situation of Figure 1(b) arises, for which we can construct the formula

$$
\delta_{G}\left(u_{2}\right)=v \bar{u}_{2} u_{1} u_{3} u_{6}\left(u_{4} \vee u_{5}\right)\left(\bar{u}_{4} \vee \bar{u}_{5}\right) \bar{u}_{4} u_{5} .
$$




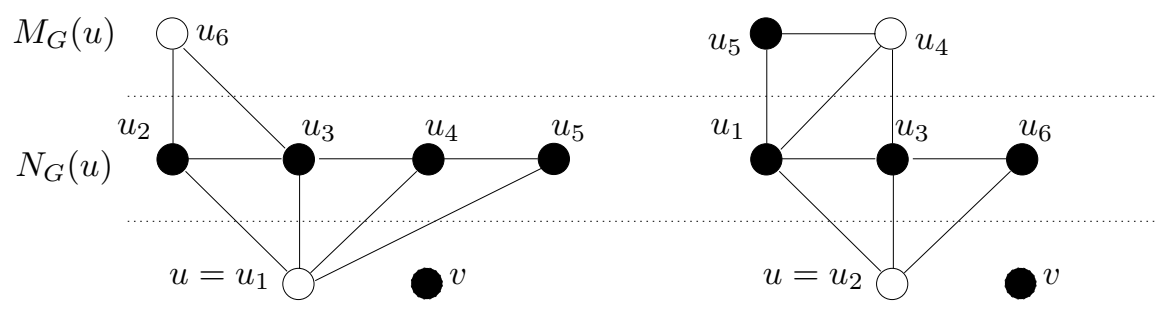

(a)

(b)

Fig. 1. Choosing $u=u_{1}$ does not give us a switch, choosing $u=u_{2}$ does.

In this case there is one selector that satisfies the formula, the one which selects $u_{5}$, but does not select $u_{4}$ (the selector also selects all other vertices except $u_{2}$ ). Applying this selector to the graph results in the simple cycle of length 7 .

\section{$5 \quad$ Bipartite switching classes}

In this section we consider the problem of determining whether a switching class contains a bipartite graph.

Starting from a bipartite graph and applying an arbitrary switch, the resulting graph is always 4-colourable (Lemma 3.30 in [7]). Hence if we start from a bipartite graph, say $G=(V, E)$ with bipartition $A, V-A$, and we switch with respect to a selector $\sigma$ then we obtain the graph $G^{\sigma}$, in which

- the sets $\sigma \cap A, \sigma \cap(V-A),(V-\sigma) \cap A$ and $(V-\sigma) \cap(V-A)$ are independent,

- the sets $\sigma \cap A$ and $\sigma \cap(V-A)$ are completely connected,

- as are the sets $(V-\sigma) \cap A$ and $(V-\sigma) \cap(V-A)$.

Note that up to three of the four sets may be empty. An example where $A \cap \sigma$ is empty is given in Figure 2 .

In the following we shall derive a 2SAT formula which specifies for each vertex, whether it must be in either the top or lower complete bipartite graph, or it may be in either one. We begin however with a small but essential lemma.

Lemma 4. A graph $G$ is complete bipartite if and only if $G$ does not have an induced $K_{3}$ nor $K_{2} \oplus K_{1}$. 
Proof. By Lemma 1, all complete bipartite graphs can be switched into the discrete graph, and these are the only graphs for which this holds.

The result on triangles by Seidel [11] states that the parities of edges in any induced subgraph on three vertices is invariant under switching and, the other way around, if two graphs (on the same vertex set) have the same parities for corresponding triangles, then they can be switched into each other.

The invariance implies that for all complete bipartite graphs, the parity of edges of $T$ is zero, as it is for the discrete graph. But the parity of edges in both $K_{3}$ and $K_{2} \oplus K_{1}$ is one, and they are the only such graphs on three vertices.

Theorem 4. Deciding that a switching class contains a bipartite graph or not can be done in time cubic in the number of vertices in the graph.

Proof. Let $G=(V, E)$ be a graph in the switching class. We start by fixing a single vertex $u \in V$ to go "up". Up refers to the top complete bipartite graph (see Figure 2) and will be represented as 0 (or, equivalently, false) in our formulas. By Lemma 4, the only thing to take care of, is to forbid subgraphs $K_{3}$ and $K_{2} \oplus K_{1}$. The formula that forbids $K_{3}$ and $K_{2} \oplus K_{1}$ in the top complete bipartite graph (assuming that $u$ is part of it) is as follows (here $V^{\prime}=V-\{u\}$ ):

$$
\begin{aligned}
u p_{G}(u)= & \bigwedge\left\{x \vee y \mid G(x u)=G(y u), G(x y)=1, x, y \in V^{\prime}\right\} \wedge \\
& \bigwedge\left\{x \vee y \mid G(x u) \neq G(y u), G(x y)=0, x, y \in V^{\prime}\right\}
\end{aligned}
$$

The first part of the formula takes care of the case that $x y$ is an edge in $G$. We then have to force one of $x$ and $y$ down to the lower part (i.e., one of them must be true). This includes both the case of $K_{3}$ and one of the three cases of an induced $K_{2} \oplus K_{1}$. The second part of the formula takes care of the case where $x y$ is not an edge in $G$, but we do have a subgraph $\left.G\right|_{\{u, x, y\}}$ isomorphic to $K_{2} \oplus K_{1}$ (these are the other two cases for $K_{2} \oplus K_{1}$ ).

The formula above only forces vertices down. This is not enough to guarantee that on the down side we always obtain a complete bipartite graph (in fact you can always choose all vertices but $u$ to go down, so pretty much every graph can arise there). To set things right we do the same as above, but now we construct a formula $\operatorname{down}_{G}(v)$ for a vertex $v$, that tells us of which pairs of vertices at least one has to go to the "up" side. This formula only differs from $u p_{G}(v)$ in the fact that every literal occurs complemented, because we have to exchange "up" with "down" 
(here $\left.V^{\prime}=V-\{v\}\right)$ :

$$
\begin{aligned}
\operatorname{down}_{G}(v)= & \bigwedge\left\{\bar{x} \vee \bar{y} \mid G(x v)=G(y v), G(x y)=1, x, y \in V^{\prime}\right\} \wedge \\
& \bigwedge\left\{\bar{x} \vee \bar{y} \mid G(x v) \neq G(y v), G(x y)=0, x, y \in V^{\prime}\right\}
\end{aligned}
$$

Finally, we can construct the 2SAT formula

$$
c_{G}(u, v)=u p_{G}(u) \wedge \operatorname{down}_{G}(v),
$$

for different vertices $u$ and $v$. In $c_{G}(u, v)$ a small optimization is possible by taking $V^{\prime}=V-\{u, v\}$ in $u p_{G}(u)$ and $\operatorname{down}_{G}(v)$.

The above reasoning should make clear that every way of satisfying the formula guarantees that the graph $G$ is split into two, arbitrarily connected, complete bipartite graphs. It does not yet guarantee that if the formula is not satisfiable, then the graph cannot be partitioned into two complete biparite graphs. This can be done by fixing $u$ and to let $v$ run through $V(G)-\{u\}$ : if a graph has a bipartite switch, then there has to be a partition in which $u$ goes up and one of the other vertices goes down. After fixing $u$ to belong to the top complete bipartite graph, we have assumed all we can, and we simply have to try all other vertices for $v$.

We have now shown how the problem of deciding whether a switching class is bipartite can be reduced to finding a solution to a linear number of 2SAT problems. Since 2SAT can be solved in time linear in the size of the formula [1], and we construct each of the formula in time quadratic in the number of vertices, we obtain an algorithm cubic in the order of the graph.

Example 2. Take the graph $G$ depicted in Figure 2. The selector $\left\{u_{4}\right\}$ gives us a bipartite graph.

The picture already implies that if we choose $u=u_{1}$ and $v=u_{4}$, then we should obtain a satisfiable formula $c\left(u_{1}, u_{4}\right)$, for which we need

$$
u p_{G}\left(u_{1}\right)=\left(u_{2} \vee u_{3}\right)\left(u_{3} \vee u_{5}\right)\left(u_{6} \vee u_{7}\right)
$$

and

$$
\operatorname{down}_{G}\left(u_{4}\right)=\left(\bar{u}_{2} \vee \bar{u}_{6}\right)\left(\bar{u}_{2} \vee \bar{u}_{5}\right)\left(\bar{u}_{3} \vee \bar{u}_{5}\right)\left(\bar{u}_{3} \vee \bar{u}_{7}\right)\left(\bar{u}_{5} \vee \bar{u}_{7}\right)
$$

Here we have given the "optimized" version. The reader can now verify that setting $u_{3}=u_{6}=1$ and $u_{2}=u_{5}=u_{7}=0$ makes $c\left(u_{1}, u_{4}\right)$ true. 


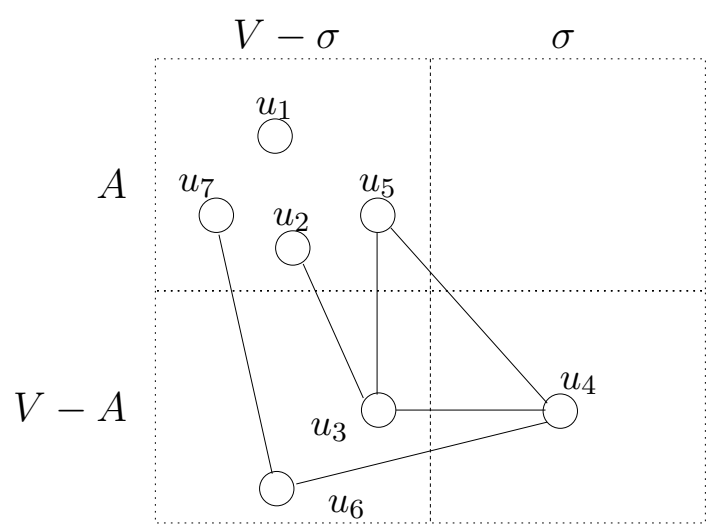

Fig. 2.

Choosing $u=u_{1}$ and $v=u_{7}$ yields a formula $c\left(u_{1}, u_{7}\right)$ which is not satisfiable. This is because both vertices are not connected to any vertex in the triangle $\left\{u_{3}, u_{4}, u_{5}\right\}$. We get

$$
u p_{G}\left(u_{1}\right)=\left(u_{3} \vee u_{4}\right)\left(u_{3} \vee u_{5}\right)\left(u_{4} \vee u_{5}\right) \ldots
$$

and

$$
\operatorname{down}_{G}\left(u_{7}\right)=\left(\bar{u}_{3} \vee \bar{u}_{4}\right)\left(\bar{u}_{3} \vee \bar{u}_{5}\right)\left(\bar{u}_{4} \vee \bar{u}_{5}\right) \ldots
$$

One of $u_{3}, u_{4}$ and $u_{5}$ must be assigned 1. Assume, without loss of generality, that this is $u_{3}$. But then $\left(\bar{u}_{3} \vee \bar{u}_{4}\right)$ implies that $u_{4}=0$. This implies that $u_{5}=1$ (because of $\left.\left(u_{4} \vee u_{5}\right)\right)$, but also that $u_{5}=0\left(\right.$ by $\left.\left(\bar{u}_{3} \vee \bar{u}_{5}\right)\right)$. The example shows that we may choose our $u$ and $v$ incorrectly. However, it is guaranteed that whatever our choice for $u$ : if the graph has a bipartite switch, then there exists a vertex $v \neq u$ for which the constructed formula is satisfiable.

\section{Some final words}

In this paper we have shown for three different graph problems that the same problem for switching classes is polynomial. In the case of bipartiteness, the result can also be used to help solve the original problems for graphs as follows.

From the above we may conclude that if $G$ has a bipartite switch, then $G$ is 4-colourable. Hence the algorithm implied in the theorem can be used as an approximative algorithm for this problem and will find a 
4-colouring in polynomial time. There is no guarantee however that the colouring of $G$ is minimal.

Not surprisingly, in view of the $\mathrm{P}$ versus $\mathrm{NP}$ conjecture, there are graphs which are 4-colourable, but which do not have a bipartite switch.

\section{References}

1. B. Aspvall, M.F. Plass, and R.E. Tarjan. A linear-time algorithm for testing the truth of certain quantified boolean formulas. Inf. Proc. Letters, 8(3):121 - 123, 1979.

2. P.J. Cameron. Cohomological aspects of two-graphs. Math. Z., 157:101 - 119, 1977.

3. D.G. Corneil and R.A. Mathon, editors. Geometry and Combinatorics: Selected Works of J.J. Seidel. Academic Press, Boston, 1991.

4. A. Ehrenfeucht, J. Hage, T. Harju, and G. Rozenberg. Complexity issues in switching of graphs. In H. Ehrig, G. Engels, H.-J. Kreowski, and G. Rozenberg, editors, Theory And Application Of Graph Transformations - TAGT'98, volume 1764 of Lecture Notes in Computer Science, pages 59-70, Berlin, 2000. Springer-Verlag.

5. A. Ehrenfeucht, T. Harju, and G. Rozenberg. The Theory of 2-Structures. World Scientific, 1999.

6. J.L. Gross and T.W. Tucker. Topological Graph Theory. Wiley, New York, 1987.

7. J. Hage. Structural Aspects Of Switching Classes. PhD thesis, LIACS, 2001. http://www.cs.uu.nl/people/jur/2s.html.

8. J. Hage and T. Harju. A characterization of acyclic switching classes using forbidden subgraphs. Technical Report 5, Leiden University, Department of Computer Science, 2000. Submitted to Siam J. Disc. Math.

9. C.L. Mallows and N.J.A. Sloane. Two-graphs, switching classes and Euler graphs are equal in number. SIAM J. Appl. Math, 28:876 - 880, 1975.

10. J.J. Seidel. Graphs and two-graphs. In Proc. 5th Southeastern Conf. on Combinatorics, Graph Theory, amd Computing, Winnipeg, Canada, 1974. Utilitas Mathematica Publishing Inc.

11. J.J. Seidel. A survey of two-graphs. In Colloquio Internazionale sulle Teorie Combinatorie (Rome,1973), volume I, pages 481-511, Rome, 1976. Acc. Naz. Lincei. Reprinted in [3].

12. J.J. Seidel and D.E. Taylor. Two-graphs, a second survey. In L. Lovasz and V.T. Sós, editors, Algebraic Methods in Graph Theory (Proc. Internat. Colloq., Szeged, 1978), volume II, pages 689-711, Amsterdam, 1981. North-Holland. Reprinted in [3].

13. T. Zaslavsky. Biased graphs. I. Bias, balance, and gains. J. Combin. Theory, Ser. B, 47:32-52, 1989 . 